\title{
Club de periodismo como incidente comunicacional en el proceso educativo
}

\author{
Journalism club as a communicational incident \\ in the educational process
}

\author{
Jefferson J. Sandoval G. \\ Universidad de Guayaquil \\ jefferson.sandovalg@ug.edu.ec \\ Código Orcid: https://orcid.org/0000-0002-3732-2041 \\ Tomás H. Rodríguez C. \\ Universidad de Guayaquil \\ tomas.rodriguezc@ug.edu.ec \\ Código Orcid: https://orcid.org/0000-0002-4673-9295
}

\begin{abstract}
Resumen
Este trabajo analiza la recepción de los géneros periodísticos interpretativos en la Academia Naval Guayaquil, a partir de la incidencia comunicacional del Club de Periodismo del diario El Telégrafo. Se buscó identificar los procesos de recepción de los géneros interpretativos y su incidencia comunicacional en los estudiantes. El tipo de investigación fue descriptiva, con una metodología hermenéutica y nueve técnicas de investigación (entre cuantitativas y cualitativas). La población para la investigación fue de 357 estudiantes, usando una técnica probabilística con ecuaciones matemáticas para cuantificar la muestra, la misma que correspondió a 145 alumnos. A través de esto, se logró observar la necesidad de implementar los géneros interpretativos como tema de estudio, para generar y desarrollar las habilidades cognitivas y diseñar estrategias de comunicación efectivas dentro de la institución, todo esto enfocado a mejorar el proceso de aprendizaje.
\end{abstract}

\section{Palabras clave}

Comunicación, educación, recepción, incidencia, géneros interpretativos.

Forma sugerida de citar: Sandoval, Jefferson y Rodríguez, Tomás (2018). Club de periodismo como incidente comunicacional en el proceso educativo. Universitas, 29, pp. 177-199. 


\begin{abstract}
In this work it was analyzed the reception of interpretive genres in Guayaquil Naval Academy based on the communicational impact of the Journalism Club from the newspaper El Telégrafo. It was sought to identify the processes of reception of the interpretive genres and their communicational incidence on students. The type of research is descriptive, the methodology is hermeneutical. In this investigation it was used nine research techniques, between quantitative and qualitative. Population is 357 students, it was used the probabilistic technique with mathematical equations to quantify the sample, which corresponds to 145 students. It was found out that there is a need to implement interpretive genres in the study area, to generate and develop cognitive skills, and design effective communication strategies in the institution. All these techniques will help to improve the learning process.
\end{abstract}

\title{
Keywords
}

Communication, education, reception, incidence, interpretive genres.

\section{Introducción}

El conocimiento de los géneros interpretativos desde el colegio, comprende un desenvolvimiento en las áreas de educación, comunicación, información y redacción de datos. Los clubes de periodismo en los centros educativos son ideados para ejercer el desarrollo de las habilidades cognitivas a temprana edad. El pensar e investigar de una manera enfática sobre problemas recurrentes de la sociedad, los invita a manifestar esas habilidades que no son desarrolladas durante la etapa de bachillerato, sino mucho tiempo después, cuando entran a una carrera universitaria; por tal motivo su implementación a escala progresiva dará forma y guía a estas destrezas.

Esta investigación promueve el análisis de recepción e incidencia que producen estos géneros en los estudiantes de la Academia Naval Guayaquil, que ha sido sede del Club de Periodismo del diario público El Telégrafo.

Esta indagación presenta un problema frecuente dentro de las unidades educativas, como por ejemplo, la no utilización de planes estratégicos que vinculen el periodismo con las demás asignaturas, lo que ocasiona futuros jóvenes sin la habilidad de conocer el panorama que los rodea o la poca es- 
timulación de sus sentidos para actuar en pro de una sociedad ávida de información como la actual.

Por tal motivo se ha formulado la siguiente pregunta: ¿Cómo se analiza la recepción de los géneros interpretativos en los alumnos de Bachillerato de la Academia Naval Guayaquil a partir de la incidencia comunicacional del Club de Periodismo de El Telégrafo?, la misma que será contestada más adelante.

Dentro del proyecto se conocen nuevos referentes teóricos que avalan este proceso, la utilización de métodos y técnicas para encontrar resultados válidos y fehacientes del progreso, asimismo, se visualiza el campo de los clubes en la actualidad y el desempeño que estos ejercen en los alumnos. No obstante, se plantea una propuesta de inserción de estos géneros a través de un plan de revista mensual realizado por los propios docentes y jóvenes.

De esta forma se expone el objetivo general, que concluye en identificar los procesos en cuanto al análisis de recepción de los géneros interpretativos y su incidencia comunicacional en los alumnos de bachillerato de la Academia Naval Guayaquil, los mismos que fueron impartidos por el Club de Periodismo de El Telégrafo en el año 2017. Se deja claro que los objetivos específicos - como el investigar las teorías, métodos y técnicas de la incidencia y recepción comunicacional, variables que se presentan a lo largo del proceso - son los mismos que ayudan a conseguir los resultados, el desarrollo del objetivo general y el planteamiento de la propuesta mencionada.

En el transcurso de este artículo se desglosa a la comunicación como una de las ciencias que permite al ser humano dar significado a su lenguaje y a la forma que transmite un mensaje. No está de más comprender que a través de un club de periodismo esta área se refuerza y se visualiza como el campo de partida que toma esta investigación. De la misma forma, se refuerza a la educación como una disciplina en la cual intervienen métodos y técnicas que facilitan la enseñanza y el aprendizaje de nuevos conocimientos. Además, se determina la predisposición de los estudiantes en cuanto a la inserción del periodismo con las demás asignaturas.

Cabe destacar que la comunicación y la educación, son fundamentales en el proceso investigativo, los mismos que al cohesionarse forman vertientes de estudio, por lo que al avanzar con el proyecto comunicativo se destaca al mismo tiempo el eje educativo la segunda:

La educación ha de ser pensada, practicada y valorada desde diferentes perspectivas. Hay que sumar las aportaciones de los diversos sectores y actores a 
la tarea educativa. La educación es un fenómeno social y cultural, y no solo un proceso instructivo y cognitivo que se lleva a cabo en las escuelas (Freinet y Saint-Luc en González, 2013, p. 16).

La fehaciente realidad de las instituciones nos demuestra que ellas proponen las mismas actividades de aprendizaje sin ningún cambio, lo que provoca desinterés por parte del estudiantado y motiva la conducta "repetitoria" que ha marcado la educación por años. Sin embargo, la inserción de dinámicas extracurriculares o la integración de estas a la malla curricular, promueven una salida de este círculo interminable de frustraciones educativas.

Este mismo proceso revela a la enseñanza como un factor imprescindible, pero que sin una comunicación efectiva se vuelve una pequeña versión de una estructura gigante, tal como puede inferirse de las opiniones de Mario Kaplún:

El profesor uruguayo Mario Kaplún (1997) usaba expresiones como: “se aprende al comunicar", "conocer es comunicar" o "del educando oyente al educando hablante", y afirmaba: "educarse es involucrarse y participar en un proceso de múltiples interacciones comunicativas” (Pérez y Valdés, 2018, p. 59).

No existe razón para no involucrarse en los procesos educativos de las instituciones, puesto que ellos necesitan una mejora en cuanto a sus estrategias comunicativas y el mismo Kaplún lo menciona: se debe participar conjuntamente y más aún con jóvenes en etapa de formación de sus habilidades. Pero la educación y los medios deben aparecer unidos desde temprana edad:

El ámbito escolar es uno de los espacios apropiados para iniciar la educación en medios. El desarrollo del pensamiento crítico y creativo y la capacidad de análisis constituyen dos grandes oportunidades de aprendizaje a partir del uso de las tecnologías de información y comunicación en el ámbito educativo, destinado a crear un periódico digital donde la escuela y sus protagonistas son generadores de noticias (Fischietto, 2014, p. 58).

Por esta razón, la educación que se imparta será vital en el desarrollo de las habilidades y construcción de los conocimientos vinculados a la relación que tiene la comunicación con esta área. Porque a través de ella se crean hábitos, valores, creencias, y no solo se aprende en un aula de clase, sino que al buscar información de internet, de personas y de algún otro sitio - lo que se conoce como educación autodidacta - , se incentiva al estudian- 
tado a permanecer en los clubes con la aportación de nuevas ideas. Por eso, un proceso de aprendizaje a temprana edad sobre la comunicación y el periodismo, sería la solución a los errores que se cometen a la hora de redactar o manifestar ideas.

Al destacar el periodismo dentro de las actividades extracurriculares, los jóvenes interactúan dentro del área comunicativa, sin embargo, desconocen la profesión y por ende asumen que la misma solo es una carga de trabajo. Es así que en el trabajo de titulación de Castro y Ortiz se puede leer lo siguiente:

Cuando se ejerce periodismo educativo, se requiere que los estudiantes desarrollen habilidades básicas para escribir, organizar los pensamientos, hacer un primer borrador y expresar ideas (editar, pulir y presentar un producto final). Esta forma de utilizar el periodismo, demanda que los estudiantes produzcan mensajes en forma de escritos (artículos de opinión, noticias, etc.); avisos publicitarios persuasivos (texto e imagen); y permite la aplicación práctica de los conceptos teóricos (Castro y Ortiz, 2011, p. 6).

El periodismo educativo o la educación periodística (a temprana edad), organizan y desarrollan el pensamiento de los jóvenes, promoviendo la creación de mensajes inéditos, el mejoramiento de las técnicas de investigación y la ampliación de conocimientos y las destrezas de escritura. El periodismo motiva a que los jóvenes se involucren en actividades que no conocían, ya no solo en la malla curricular, sino que visualicen los problemas comunes y desatendidos de la sociedad, donde ellos puedan tomar parte de los inconvenientes y a través de la redacción aprendan a contar o expresar las situaciones que los rodean. Se trata de un conocimiento indispensable para el desarrollo de cualquier otra asignatura, como se puede ver a continuación:

Con esta concepción nace el modelo "aprender haciendo", configurando el carácter activo-participativo de la formación periodística, el mismo que involucra el planteamiento de problemas auténticos para estimular el pensamiento, las observaciones, las situaciones analíticas y las oportunidades para descubrir soluciones. De esta manera nace el Club de Periodismo de Diario El Telégrafo, una iniciativa que consolida la redacción como el eje principal del aprendizaje periodístico y la mejor manera de aprender a escribir es promoviendo la práctica constante como medio de conocer, analizar, valorar y criticar (Correa, 2016, p. 17). 
Fue así como el club de periodismo surgió desde los estudiantes para los estudiantes. Cabe recalcar que todos los jóvenes fueron guiados por profesionales de la comunicación, pero con el objetivo no de enseñar la profesión, sino de generar un pensamiento crítico. En un principio, solo se lo analizó como una ayuda a los estudiantes universitarios para la expansión de sus prácticas o una ampliación de su vida laboral, sin embargo, la aceptación fue tal, que se lo transmitió a los colegios con el afán de mejorar el método de aprendizaje utilizado en el pensum académico.

El porqué de los géneros interpretativos se explica por el proceso cognitivo del estudiante: a través de la redacción de los textos incrementa o perfecciona su creatividad, su formación y despliegue de ideas, así como su desenvolvimiento en las escenas periodísticas, sobre todo al utilizar el conocimiento aprendido en las demás asignaturas.

Dentro de este análisis se tuvieron en cuenta dos variables que permitieron medir la opinión y el desempeño de los estudiantes. La primera se basó en la recepción de los géneros interpretativos como un sistema de enseñanza y de mejoramiento en el desempeño estudiantil. La segunda fue la incidencia que provoca recoger esta información, adoptarla, transformarla y utilizarla en los diferentes campos de estudio en los que se desenvuelven los jóvenes. Porque esto repercute tanto en su vida social como escolar.

\section{Recepción comunicacional}

Como primer punto del análisis, se trabaja la estructura de la recepción que se origina dentro de la comunicación. Asimismo, se contempla que esta permite acoger información, interpretarla y sacar de ella un estímulo capaz de generar una retroalimentación del investigador y de los estudiantes. Un proceso científico valedero de analizar en el documento Estudio de recepción del periódico comunitario El Chulla Quiteño, deja claro que:

El análisis de la recepción es una corriente teórica de las ciencias de la comunicación que ofrece interesantes posibilidades para la investigación; como marco teórico y fuente de conceptos útiles para comprender la relación que establecen las audiencias con los medios y como una fértil matriz de recursos metodológicos y técnicas para producir información sobre dicha relación (Flores en Tapia, 2016, p. 17). 
En este sentido, se propone un campo de investigación que va en relación hacia el aprendizaje de los jóvenes, de cómo captan las imágenes, las ideas y sobre todo el conocimiento, fundamental en su proceso de formación, progreso y construcción de habilidades. Por eso la recepción abarca un proceso fundamental en este planteamiento, ayuda a cuantificar y medir el desarrollo de cada uno de los temas propuestos en el club de periodismo. Además, se estimulan los sentidos, la proyección de los escritos, el trabajo continuo de los estudiantes y el carácter periodístico que se necesita para trabajar en un medio.

Así, es necesario reconocer las diferentes formas en que los estudiantes aprenden, para lo cual es de utilidad la siguiente tabla de La "Flipped Classroom" como recurso metodológico aplicado a la docencia:

\section{Tabla 1}

\section{Dimensiones del aprendizaje}

\begin{tabular}{|l|l|l|l|}
\hline $\begin{array}{c}\text { Aprendizaje } \\
\text { Significativo }\end{array}$ & $\begin{array}{c}\text { Clasificación de las } \\
\text { relaciones entre los } \\
\text { conceptos }\end{array}$ & $\begin{array}{c}\text { Enseñanza } \\
\text { audiotutelar bien } \\
\text { diseñada }\end{array}$ & $\begin{array}{l}\text { Investigación científica } \\
\text { o de creación (música o } \\
\text { arquitecturas nuevas) }\end{array}$ \\
\hline & $\begin{array}{l}\text { Conferencias, o } \\
\text { presentaciones de la mayor } \\
\text { parte de los libros de texto }\end{array}$ & $\begin{array}{l}\text { Trabajo escolar en el } \\
\text { laboratorio }\end{array}$ & $\begin{array}{l}\text { "Investigación" más } \\
\text { rutinaria o producción } \\
\text { intelectual }\end{array}$ \\
\hline $\begin{array}{l}\text { Aprendizaje } \\
\text { por repetición }\end{array}$ & Tablas de multiplicar & $\begin{array}{l}\text { Aplicación de } \\
\text { fórmulas para } \\
\text { resolver problemas }\end{array}$ & $\begin{array}{l}\text { Soluciones a } \\
\text { rompecabezas por } \\
\text { ensayo y error }\end{array}$ \\
\hline & $\begin{array}{l}\text { Aprendizaje por } \\
\text { recepción }\end{array}$ & Guiado & Autónomo \\
\cline { 2 - 4 } & Aprendizaje por descubrimiento \\
\hline
\end{tabular}

Fuente: Ausubel et al., en Balbas, 2014,p. 11

En esta tabla se hace énfasis a los distintos métodos de aprendizaje entre los cuales están el significativo, por repetición, por descubrimiento y por recepción - que engloban los procesos y técnicas utilizadas por docentes para el sistema educativo, no obstante, se plantea un estudio comunicacional donde el estudiante a más de recibir la clase, formule ideas relevantes de lo que ha captado durante todo el tiempo.

No hay que olvidar que cada metodología aplica un sinnúmero de factores entre los estudiantes, las mismas que desarrollan habilidades, entre ellas, 
las que benefician el perfeccionamiento de la comunicación, como muestra se refleja la participación continua de los asistentes, es decir, una retroalimentación entre ambos bandos.

\section{Incidencia del proceso comunicacional}

Por otro lado, la incidencia se posiciona desde el punto de vista que logra influenciar o repercute en la vida social de un joven. Dentro del aula de clase, los jóvenes son expuestos a decenas de conocimientos, sin embargo, no todos ellos pueden ser adquiridos. En el club de periodismo de El Telégrafo se propone la construcción y exposición de los géneros interpretativos, y la repercusión en el aprendizaje de los estudiantes; además, dentro de la investigación intervienen otros factores como la motivación y la participación, que también pueden ser valorados.

Es importante mencionar que la Incidencia de la Metodología en el Rendimiento Académico de la Matemática los alumnos utilizan desde los primeros niveles educativos la memoria y la ejercitación repetitiva para estudiar. A esto se suma que la mayoría de los estudiantes llegan con grandes falencias, entre ellas, falta de conocimientos básicos mínimos en las diferentes disciplinas, carencia de hábitos de lectura y de perseverancia y esfuerzo en la búsqueda de resultados (Mena et al., en Toledo y Balseca, 2013, p. 11).

Así como en las matemáticas, que es una ciencia exacta, el lenguaje o la comunicación debe darse de una forma clara, concisa y precisa. Esto permite que el receptor, en este caso el estudiante, reciba un mensaje sin intervenciones ni modificaciones, lo que a su vez representa un proceso comunicacional superior.

Los estudiantes manejan un lenguaje básico, en ellos se refleja una carencia de conocimientos en cuanto a la redacción de una noticia, así como en la locución de pequeños textos y la realización de preguntas simples, por lo tanto, observar su determinación en las charlas propone el tema a investigar y plantear la evolución que tendrán en el transcurso del club.

Por ello, es prometedora esta investigación, como se aborda el siguiente gráfico: 
Figura 1

Categorías fundamentales
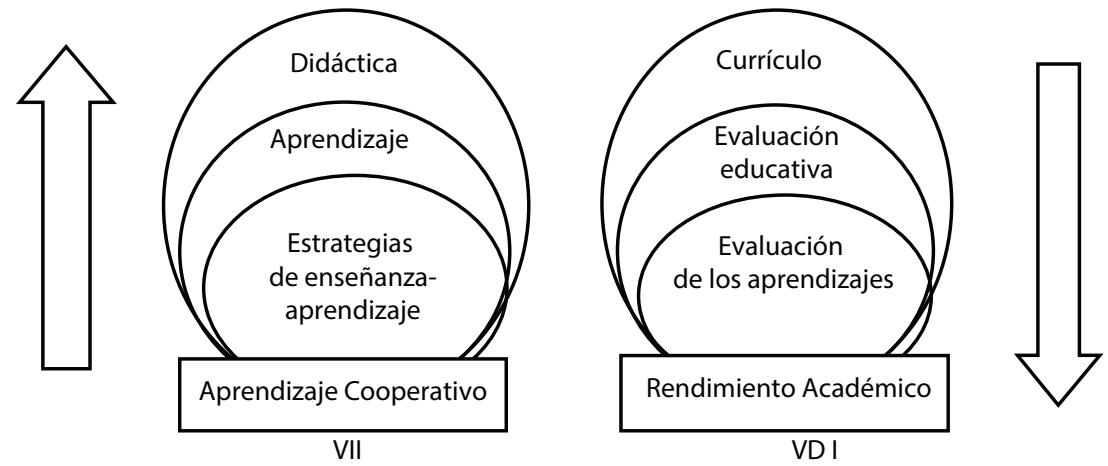

Fuente: Cotallat y DT-Núñez, 2012, p. 18

En la figura se observan dos perspectivas, la primera que apunta al aprendizaje cooperativo y cómo es transmitido a los estudiantes; por consiguiente, se plantea el uso de estrategias en la enseñanza y el método didáctico por el cual son transferidos. La segunda, producto de un procedimiento más elaborado, analiza la incidencia que tienen los conocimientos impartidos, resolviendo a través de una evaluación que mide el entusiasmo, la motivación, etc., así como cuantifica el conocimiento adquirido a través de otra valoración.

Cada área de la comunicación representa una estructura organizada y sistematizada desde el punto de vista de un periodista, por esta razón, conocer los diferentes esquemas y bases teóricas sobre la recepción e incidencia que marcan esta investigación, será relevante para ponerlos en práctica con los estudiantes.

\section{Habilidades cognitivas}

En el transcurso de esta investigación vimos que el aprendizaje periodístico genera en los estudiantes habilidades cognitivas, pues abarca el estudio del pensamiento, del lenguaje y del conocimiento. Además, apreciamos que 
la recolección de información - que en este caso son los géneros interpretativos - ayuda al proceso de creación e imaginación. Entre las habilidades a relucir se encuentra: la atención, la comprensión, la elaboración y la memorización, que son técnicas de estudio, cada una de ellas inmersas en el ser humano, pero no formadas ni desarrolladas en el proceso educativo, lo que a través del club de periodismo como una actividad extracurricular se intenta motivar. Por eso es ilustrativa la siguiente tabla del trabajo "La enseñanza de estrategias de aprendizaje en educación infantil":

Tabla 2

Habilidades de procesamiento

\begin{tabular}{|l|l|}
\hline \multicolumn{1}{|c|}{ Habilidades } & \multicolumn{1}{c|}{ Sub-habilidades } \\
\hline Observar & Auto-observación, observación directa \\
\hline Comparar & Análisis comparativo, búsqueda eficaz de información \\
\hline Ordenar, clasificar & Orden: serial, temporal, espacial \\
\hline Representar & Representación: gráfica, icónica, verbal, gestual \\
\hline Memorizar & Codificación cognitiva, evocación, reconocimiento, reconstrucción \\
\hline Evaluar & Toma de decisiones, demostración \\
\hline Transferir & Inferir, transferir, interpretar \\
\hline
\end{tabular}

Fuente: Nisbet et al., en Jiménez et al., 2007, p. 9

Es seguro precisar múltiples habilidades y sub habilidades necesarias para aprender y pensar, puesto que todas ellas están relacionadas dentro de la comunicación. Conocer las estrategias que demanda un trabajo periodístico no solo ayuda a su desarrollo intelectual, sino también a su parte locuaz. Un comunicador debe poder observar los hechos de una manera diferente a los demás, excavar lo profundo de un tema al punto de revelar nueva información que en el futuro pueda utilizar; sin embargo, estas habilidades se construyen desde la escuela y ya en el colegio su perfeccionamiento debe acelerarse. Si bien esta es una tarea muy complicada, se la puede llevar a cabo mediante técnicas de estudio como las que se expresan a continuación: 
Dentro de la Taxonomía de Bloom las habilidades cognitivas son las destrezas que permiten al individuo adquirir y desarrollar pensamiento y conocimientos nuevos. Las habilidades cognitivas se pueden clasificar en dos órdenes: las básicas y las superiores, entendiéndose con ello que las primeras facilitan la adquisición del conocimiento y las segundas la calidad y la aplicación del mismo (Mendoza et al., 2013, p. 31).

Bloom hace énfasis en la clasificación de las habilidades. Primero la persona debe adquirir conocimiento, debe poder comprenderlo y a la vez tener almacenado todos esos datos, pero nada de ello es posible si tiempo después no son utilizados, no obstante, en este análisis, las habilidades no solo mejoran, sino que muchas veces se crean.

\section{Material y métodos}

Esta investigación presenta un diseño no experimental transeccional, puesto que el análisis que se plantea dentro de la Academia Naval Guayaquil sirve para ahondar en la problemática, así como en la recolección de datos y el perfeccionamiento de las habilidades periodísticas. Igualmente, se acogió el tipo de investigación descriptiva porque la recepción e incidencia se manifiestan como las variables dentro del análisis y repercuten en sus habilidades cognitivas y el lenguaje periodístico que adquieren a través del tiempo. Se deja claro que la indagación atravesó un estudio exploratorio, que conservó las variables y dimensiones antes escritas, para concluir en una investigación descriptiva, analizando la comprensión lectora de los estudiantes, así como su construcción periodística en las aulas.

En consecuencia, se planteó la siguiente hipótesis: el desinterés en temas de periodismo por parte de los alumnos de bachillerato de la Academia Naval Guayaquil, se ve afectado por el desempeño de sus docentes en temas de noticias y el no planteamiento de proyectos periodísticos eficaces dentro de la institución. Y luego de ello, se concretó que las variables dentro del proyecto son la recepción e incidencia del proceso comunicacional, y a través de la ya mencionada hipótesis, se detalla a continuación el cuadro de la definición operacional de las variables que se utilizó por completo dentro del trabajo (en el presente artículo solo se toman las principales): 
Tabla 3

Definición operacional de las variables

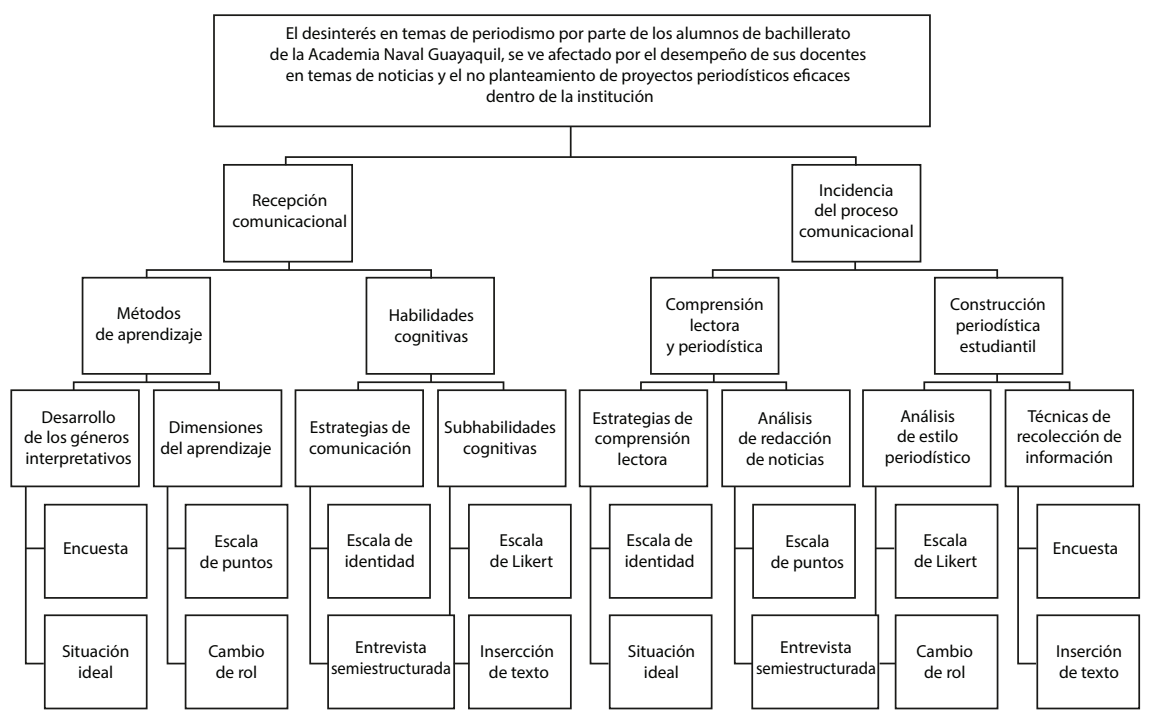

Fuente: los autores

La investigación se hizo con una metodología hermenéutica porque la relación del investigador con los alumnos se concentró en los procesos históricos de la institución y los proyectos básicos de periodismo generados a través del tiempo. Por tanto, se estructuró nueve diferentes técnicas de investigación, para observar el grado de profundidad con el que se abordó a los estudiantes de bachillerato de la Academia Naval Guayaquil. Entre ellas, cuatro fueron cuantitativas - la encuesta, escala de Likert, escala de intensidad y escala de puntos - y cinco cualitativas - investigación bibliográfica, entrevista semi estructurada, inserción de texto, cambio de rol y situación ideal-.

La población de esta indagación fue de 357 estudiantes fragmentados en doce bachilleratos unificados (primero, segundo y tercero), de la Academia Naval Guayaquil sección matutina. Por esto se debió utilizar una técnica probabilística para cuantificar la muestra de trabajo, donde las ecuaciones matemáticas complementaron el desarrollo y se estableció el número de 
estudiantes a encuestar. Así, después de la fórmula $n=(Z)^{2}(P)(Q)(n) /(E)^{2}$ $(\mathrm{N}-1)+(\mathrm{Z})^{2}(\mathrm{P})(\mathrm{Q})$, y con la tipología estratificada, se obtuvo una muestra de 145 estudiantes que se dividieron en 54 hombres y 91 mujeres.

\section{Análisis y resultados}

Las preguntas y resultados que se detallan a continuación son una recopilación de las mejores derivaciones que se manifestaron en el trabajo de campo en la Academia Naval Guayaquil. Las preguntas reflejan el estudio de las variables, dimensiones, indicadores y técnicas planteadas en el trayecto, lo que reveló resultados fehacientes y contundentes de la hipótesis. Cabe recalcar que cada pregunta fue pensada y creada desde el trabajo de investigación a través de expresiones de autores y pensamientos detallados en artículos.

\section{Figura 2}

\section{La enseñanza de los géneros interpretativos}

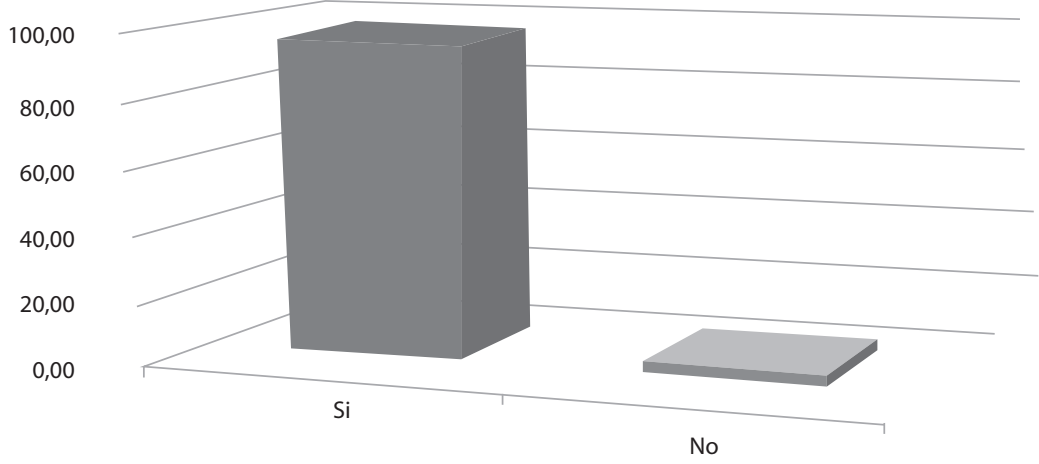

Fuente: los autores

Variable: recepción comunicacional

Dimensión: métodos de aprendizaje.

Indicador: desarrollo de los géneros interpretativos

Técnica cuantitativa: encuesta 
Pregunta 1: ¿Cree que es necesario la enseñanza de los géneros interpretativos en las aulas?

Se presta atención a la rotunda afirmación en la integración de los géneros interpretativos en el aula de clase, con un resultado del 97\%, es decir, 140 estudiantes del total de encuestados mantienen una total disposición a la inserción de un capítulo diferente en sus enseñanzas.

\section{Figura 3 \\ Manejo las dimensiones del aprendizaje}

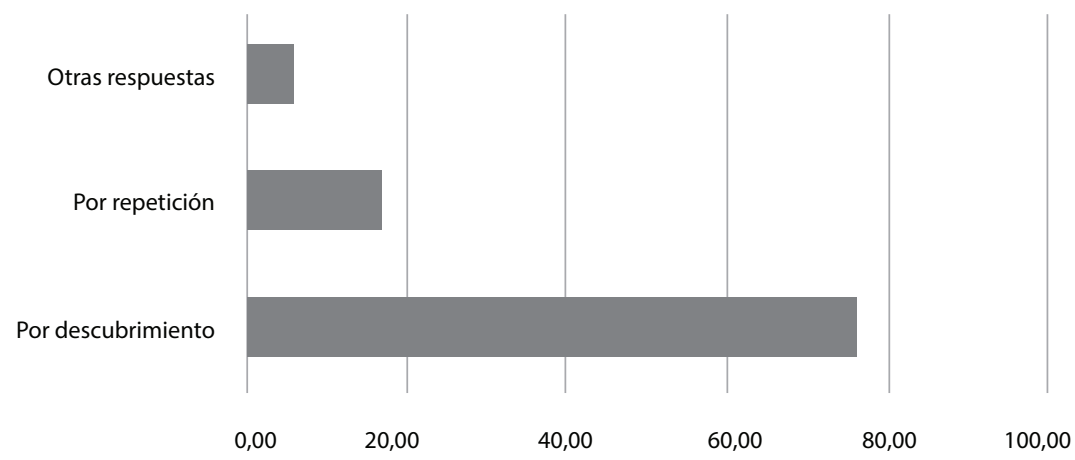

Fuente: los autores

Variable: recepción comunicacional

Dimensión: métodos de aprendizaje

Indicador: dimensiones del aprendizaje

Técnica cualitativa: cambio de rol

Pregunta 2: Si usted manejara las dimensiones del aprendizaje, ¿utilizaría el aprendizaje por descubrimiento o por repetición?

Los estudiantes reflejaron su compromiso al aprendizaje por descubrimiento con un $77 \%$ a favor, mientras que el $17 \%$ decidieron optar por la repetición. Aunque las dos dimensiones son esenciales para la enseñanza, no cabe duda que cuando se descubre una información el cerebro la guarda por mucho más tiempo y es lo que en el periodismo funciona mejor. 
Figura 4

Dimensiones del aprendizaje que impulsan el club

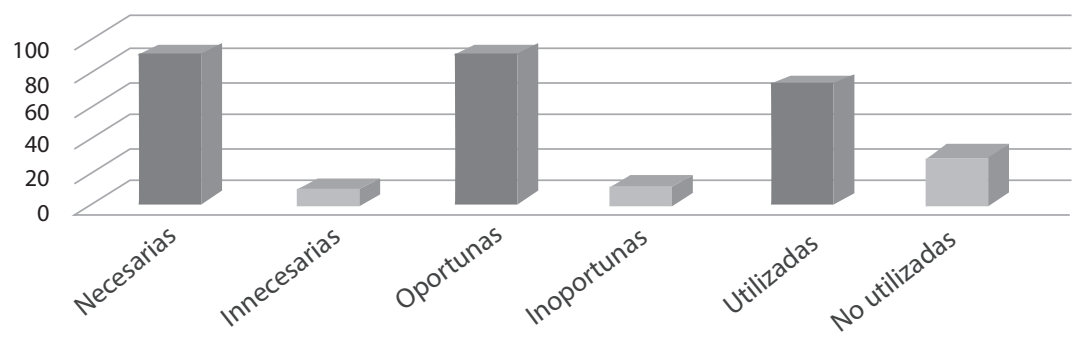

Fuente: los autores

Variable: recepción comunicacional

Dimensión: métodos de aprendizaje

Indicador: dimensiones del aprendizaje

Técnica cuantitativa: escala de puntos

Pregunta 3: Las dimensiones del aprendizaje para impulsar el club de periodismo dentro de la institución son...

Con respuestas más allá del $70 \%$ sobre impulsar un club de periodismo en la institución, los jóvenes insistieron en que su implementación es necesaria y oportuna, sin embargo, con la existencia de un club de periodismo en la institución, un $28 \%$ cree que no es utilizado correctamente, de ahí la razón de ser de esta investigación.

Figura 5

\section{Desarrollar habilidades cognitivas}

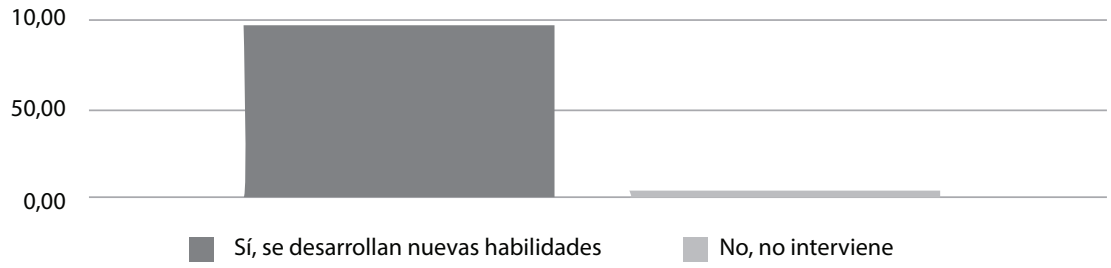

Fuente: los autores 
Variable: recepción comunicacional Dimensión: habilidades cognitivas Indicador: sub-habilidades cognitivas Técnica cualitativa: inserción de texto

Pregunta 4: Después de leer la cita, ¿cree usted que mediante un club de periodismo se puedan desarrollar habilidades cognitivas?

La cognición es una serie de procesos interrelacionados mediante los cuales obtenemos y utilizamos conocimientos relacionados con el mundo. Abarca el pensamiento, el aprendizaje, la percepción, el recuerdo y la comprensión. Por lo tanto llamaremos desarrollo cognoscitivo al crecimiento y perfeccionamiento de estos procesos y habilidades (Allueva et al., 2015, p. 2).

Una cita es el pensamiento de un autor, los estudiantes observaron y analizaron dichas palabras y el 95,86\% aseguraron que la implementación de un club de periodismo es necesario, porque a través de este se incrementan sus habilidades, lo cual no es visto por otras materias y desencadena un sinnúmero de respuestas favorables hacia la iniciativa.

\section{Figura 6 \\ Comunicación y los temas periodísticos}

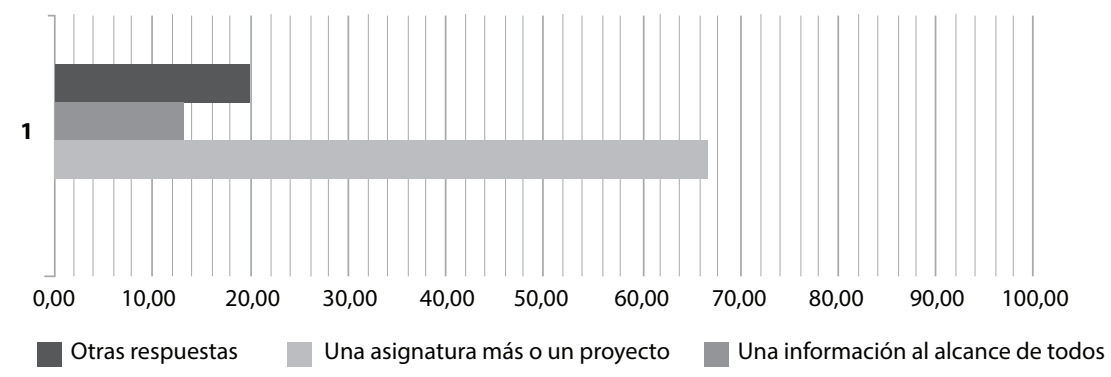

Fuente: los autores

Variable: incidencia del proceso comunicacional

Dimensión: comprensión lectora y periodística

Indicador: estrategias de comprensión lectora

Técnica cualitativa: situación ideal 
Pregunta 5: Para que la comunicación y los temas periodísticos sean enseñados de manera correcta en la institución, la comprensión de la lectura debe proponerse como...

El pilar fundamental de un buen periodista se basa en la lectura, su comprensión y el análisis que ejercen los valores fundamentales en la profesión. De esta manera, el 66,9\% expresó que si se convierte en una materia o un proyecto, se originaría un mayor desenvolvimiento en otras áreas de trabajo y no solo en el periodismo. Asimismo, se visualizó que el 13,1\% lo preferiría por estar al alcance de todos con nueva información.

Figura 7

\section{Enseñanza de redactar noticias}

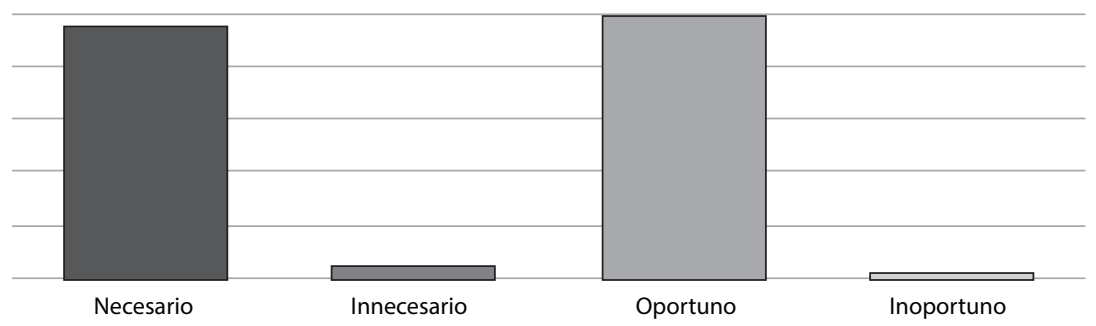

Fuente: los autores

Variable: incidencia del proceso comunicacional

Dimensión: comprensión lectora y periodística

Indicador: análisis de redacción de noticias

Técnica cuantitativa: escala de puntos

Pregunta 6: la enseñanza de redactar noticias es...

Es fácil comprender que una enseñanza pronta de los géneros periodísticos en los estudiantes es necesaria en un $95,17 \%$ y oportuna en un $98,62 \%$. De aquí su lenguaje aumenta y su pensamiento los hace ser más críticos, sin embargo, es fundamental que se lo ejerza con un poco más de rigurosidad en la institución, debido a la poca intención de fomentar esta disciplina en el aula de clase. 
Figura 8

Técnicas para el aprendizaje periodístico

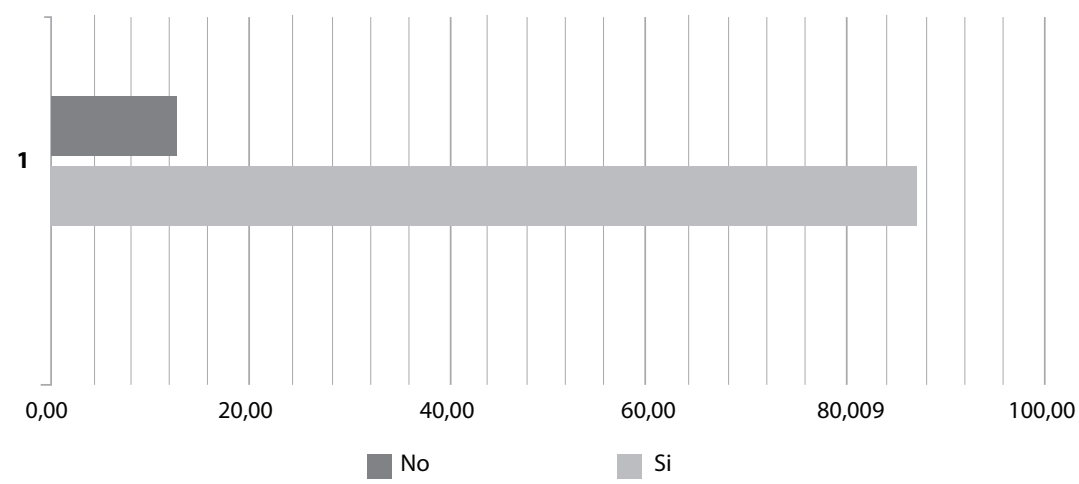

Fuente: los autores

Variable: incidencia del proceso comunicacional

Dimensión: construcción periodísticas estudiantil Indicador: técnicas de recolección de información Técnica cuantitativa: encuesta

Pregunta 7: ¿Cree usted que conocer las técnicas de recolección de información son vitales para el aprendizaje periodístico?

Como se sabe, el periodismo implica investigación, por ende, las técnicas de recolección de información abarcan un eje fundamental en este proceso. Un $87 \%$ de los estudiantes manifiestan que aprender las técnicas de recolección de información son la clave para el periodismo, pero el fin no es solo trabajar para ese cargo, sino que vaya más allá de ello, por ejemplo, con las otras asignaturas cuando son enviados a investigar y solo se concentran en una fuente, ignorando que existe un sinfín de voces que pueden ser tomadas en cuenta. De ahí la práctica e inserción de estas técnicas. 
Figura 9

Implementación de las técnicas de recolección de información

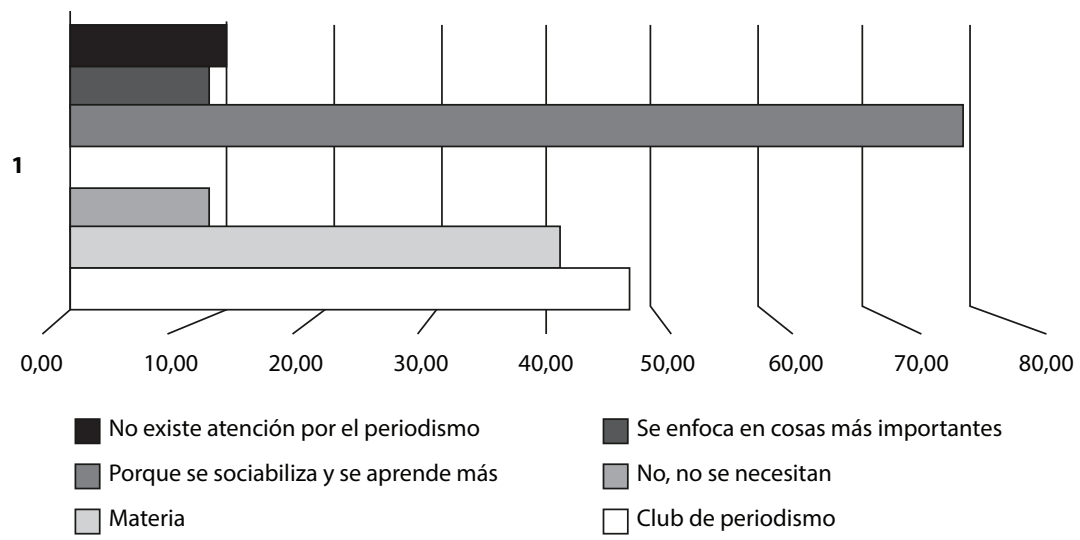

Fuente: los autores

Variable: incidencia del proceso comunicacional Dimensión: construcción periodísticas estudiantil Indicador: técnicas de recolección de información Técnica cualitativa: inserción de texto

Pregunta 8: después de leer la cita, ¿cree usted que las técnicas de recolección de información, deben ser implementadas en las aulas a través de las materias, o a partir de un club de periodismo? ¿Por qué?

Las técnicas de recolección de datos se refieren a los procedimientos para obtener datos o información. De acuerdo con Arias (2006) “...la aplicación de una técnica conduce a la obtención de información que debe ser registrada en un medio de manera que los datos puedan ser recuperados, procesados, analizados e interpretados posteriormente" (Marcano et al., 2013, p. 55).

Tras una contemplación de las palabras de un autor, se reflejó una división en cuanto a dónde enseñar las técnicas de recolección de información, se da en la inserción del periodismo como una materia en un 41,38\%, así como dentro del club de periodismo con un 46,9\%. Pero cuando se les pre- 
gunta por qué debería ser en alguna de ellas, se inclinan hacia el club con un $75,17 \%$ porque se sociabiliza y se obtiene información más específica; además, implica menos estrés como manifiesta uno de ellos y así cultivan una profunda guía hacia la carrera, pues varios alumnos se encuentran indecisos en la elección de la misma cuando salen de la unidad educativa.

\section{Conclusiones}

El presente trabajo de investigación denotó la predisposición de los estudiantes en el proceso de las técnicas de investigación y la inserción de los géneros interpretativos como una opción de integración a su malla curricular. Se concluye que la indagación formó parte de un modelo de reestructuración y construcción de conocimiento sobre el periodismo en los jóvenes de la Academia Naval Guayaquil.

Las teorías de recepción y de incidencia comunicacional son viables y desempeñan parte fundamental de los procesos efectuados en el transcurso de la investigación y recolección de datos. Con un 96,55\% del total de la muestra, se observó que la implementación de los géneros interpretativos, correspondientes a una enseñanza periodística, son viables para su inserción, así como aplicar estas dimensiones en el aprendizaje para desarrollar la comunicación y el interés hacia estos temas $(97,24 \%)$.

Al seguir con el análisis, se observa que los métodos de aprendizaje son fundamentales para la inserción de temas periodísticos y el desarrollo crítico de los estudiantes, así como un desempeño óptimo en las demás materias. Además, se plantea que la institución y los docentes manejan las estrategias de comunicación en ciertas áreas y no se toma la debida importancia de este tema, esto se ve en un 67,59\%. Mientras que los clubes de periodismo como idea formadora de las capacidades y habilidades cognitivas en los estudiantes tienen un apoyo del 95,86\%. Por eso se concluye que las habilidades cognitivas permiten al investigador conocer el proceso en el que está el estudiantado, para así promover el uso de estrategias que mejoren la comunicación dentro de las aulas.

Asimismo, la formación periodística estudiantil puede incidir en el desempeño y desenvolvimiento de las capacidades académicas, para generar un estilo dentro de esta área y consecutivamente orientar a los alumnos a una carrera universitaria. Sin embargo, el no planteamiento de proyectos dentro 
de la institución también es cuestionado por el 95,17\%. En cuanto a la redacción de noticias, los estudiantes apostaron a la necesidad de que exista esta oportunidad, pero su decisión está basada en ciertas respuestas, como en la integración a través de una asignatura o un proyecto, con un 67,5\% a favor, lo que significaría para ellos algo novedoso e interesante.

La investigación dentro del periodismo es fundamental, sin embargo, también lo es para cualquier materia. Un gran porcentaje de estudiantes no conoce las técnicas de investigación, no obstante, el 87,59\% asegura que es imperativo para el aprendizaje periodístico. Es decir, no existe una implementación en el área de la comunicación dada por los docentes. Por esto, al increparles a los jóvenes cómo deberían ser transmitidas, más de la mitad objetó que se llevara a cabo mediante un club de periodismo, porque el $75,17 \%$ cree que se enfocaría más al asunto y se aprendería a sociabilizar, aunque en la institución existe un precedente de club, el mismo debe ser mejorado y actualizado para el estudio de temas más importantes y relevantes, además de una integración colectiva entre ellos mismos.

Por último, se deja clara la integración de la comunicación y el proceso educativo. Los clubes de periodismo son esenciales tanto en las escuelas como en los colegios. Los estudiantes precisan una nueva forma de conseguir información de tal manera que más adelante se tengan jóvenes con capacidades críticas e investigativas, las cuales no son enseñadas ni clarificadas en las materias. Por eso la implementación a temprana edad de estos clubes es imprescindible.

\section{Bibliografía}

Allueva, S., Pueyo, M. y Altemir, I. (2015). Habilidades cognitivas visuales en niños con antecedentes de prematuridad (Tesis de pregrado). Universidad Zaragoza, España. Recuperado de https://goo.gl/p2bab5/

Balbas, F. (2014). La "Flipped Classroom" como recurso metodológico aplicado a la docencia de Expresión Gráfica de $4^{\circ}$ de ESO (Tesis de maestría). Universidad de Valladolid, España. Recuperado de https://goo.gl/irr5Y1/

Castro, M. y Ortiz, A. (2011). Importancia del periodismo educativo en la formación de los niños y niñas de la escuela fiscal mixta Dr. Carlos Moreno Arias del cantón Milagro (Tesis de pregrado). Universidad Estatal de Milagro, Ecuador. Recuperado de https://goo.gl/y4X6Bk/ 
Correa, D. (2016). Club de periodismo, modelo de práctica educativa para fortalecer la escritura periodística. INNOVA Research Journal, 1(6), 14-26. Recuperado de https://goo.gl/tEYR4W/

Cotallat, J. y DT-Núñez, M. (2012). Aprendizaje cooperativo y su incidencia en el rendimiento académico de los estudiantes de segundo año de Bachillerato especialidad Ciencias del Instituto Tecnológico Pelileo (Tesis de maestría). Universidad Técnica de Ambato, Ecuador. Recuperado de https:/goo.gl/sPFdnK/

Fischietto, A. (2014). Desarrollo de Periódicos Escolares en Ambiente Virtual. Revista Científica EduWeb, 8(1), 57-70. Recuperado de https://bit. 1y/2L4KVJ3/

González, J. (2013). Célestin Freinet, la escritura en libertad y el periódico escolar. Un modelo de innovación educativa en la primera mitad del siglo 20. História da Educação, 17(40), 11-26. Recuperado de https://bit. ly/2nGVHMn/

Jiménez, L., Pérez, H. y Fernández, S. (2007). La enseñanza de estrategias de aprendizaje en educación infantil. Profesorado, Revista de currículum y formación de profesorado, 11(2). Recuperado de https://goo.gl/mBbvSZ/

Marcano, M., Quintero, A. y Rodríguez, N. (2013). Plan de formación en tecnologías de información y comunicación para el profesorado de educación media del instituto escuela. Píxel-Bit, revista de medios y educación, (42), 51-64. Recuperado de https://goo.gl/f8jm93/

Mendoza, L., Zermeño, M. y Zermeño, R. (2013). Desarrollo de habilidades cognitivas y tecnológicas con aprendizaje móvil. Revista de Investigación Educativa de la Escuela de Graduados en Educación, 3(6), 30-39. Recuperado de https://goo.gl/KWLoEf/

Pérez, Z. y Valdés, A. (2018). Estrategias para la formación profesional en periodismo radiofónico: experiencias y propuestas/Strategies for professional training in radio journalism: experiences and proposals. Revista de Comunicación de la SEECI, (46), 53-63. Recuperado de https://bit. ly/2L3uBbw/

Tapia, L. (2016). Estudio de recepción del periódico comunitario El Chulla Quiteño (Tesis de pregrado). Universidad Politécnica Salesiana, Ecuador. Recuperado de https://goo.gl/TK3joo/

Toledo, D. y Balseca, C. (2013). El uso de las aulas virtuales y su incidencia en el Rendimiento Académico Estudiantil en Matemática de la Facultad de Fi- 
losofía, Letras y Ciencias de la Educación de la Universidad Central del Ecuador (Tesis de maestría). Universidad Técnica de Ambato, Ecuador. Recuperado de https://goo.gl/gscgBa/

Fecha de recepción: 2018/02/20; Fecha de aceptación: 2018/08/15;

Fecha de publicación: 2018/09/01 